\title{
Elementos relacionales del capital psicológico y cambio organizacional en empleados del sector terciario de la cuidad de Bogotá - Colombia ${ }^{1}$
}

\author{
Relational elements of psychological capital and organizational change in \\ employees of the tertiary sector in the city of Bogotá - Colombia
}

\section{Elementos relacionais da capital psicológico e mudança organizacional nos trabalhadores do sector terciário da cidade de Bogotá - Colômbia}

\author{
Mónica García Rubiano ${ }^{2}$ \\ Carlos Forero Aponte ${ }^{3}$
}

Recibido: 15.01.2017 - Arbitrado: 16.02.2017 - Aprobado: 14.03.2017

\section{Resumen}

El propósito de esta investigación fue determinar la relación entre cambio organizacional y el constructo capital psicológico con sus cuatro componentes en trabajadores de dos empresas de la ciudad de Bogotá, con la participación de 100 colaboradores. Es una investigación no experimental bajo un estudio transversal correlacional. Los resultados confirmaron las seis hipótesis propuestas y muestran correlaciones positivas significativas entre el capital psicológico en sus cuatro componentes y el cambio organizacional.

Palabras clave: capital psicológico, cambio organizacional, optimismo, resiliencia, esperanza, autoeficacia.

\begin{abstract}
Abstrac
The purpose of this research is to determine the relationship between organizational change and the psychological capital construct with its four components in workers from two companies in the city of Bogotá, with the participation of 100 employees. It is a nonexperimental investigation under a correlational cross-sectional study. The results confirmed the six proposed hypotheses and show significant positive correlations between psychological capital, its four components, and organizational change.

\footnotetext{
${ }^{1}$ Articulo presenta resultados de investigación denominada "Proyecto cambio y capital psicológico", realizada en la Universidad Católica de Colombia.

${ }^{2}$ Magister en psicología, Universidad Católica de Colombia; Docente, Universidad Católica de Colombia, Bogotá, Colombia,mgarcia@ucatolica.edu.co

${ }^{3}$ Magister en psicología, Universidad Católica de Colombia; Docente, Universidad Católica de Colombia, Bogotá, Colombia, cforero@ucatolica.edu.co
}

Citación del artículo: García, M., Forero, C. (2017). Elementos relacionales del capital psicológico y cambio organizacional en empleados del sector terciario de la cuidad de Bogotá - Colombia. Revista Katharsis, N 23, enero-julio 2017, pp.129-141, Disponible en http://revistas.iue.edu.co/index.php/katharsis 
Keywords: psychological capital, organizational change, optimism, resilience, hope, selfefficacy.

\section{Resumo}

O objetivo desta pesquisa foi determinar a relação entre a mudança organizacional e construir capital psicológico com seus quatro componentes em trabalhadores de duas empresas na cidade de Bogotá, com a participação de 100 empregados. É uma pesquisa não-experimental no âmbito de um estudo transversal, correlacional. Os resultados confirmaram as seis propostas hipóteses e mostram correlações positivas significativas entre capital psicológico, seus quatro componentes e mudança organizacional.

Palavras-chave: capital psicológico, mudança organizacional, otimismo, resiliência, esperança, auto-eficácia.

\section{Introducción}

\section{Capital psicológico}

De acuerdo con Luthans, Avolio, Avey \& Norman (2007), el capital psicológico se relaciona con el comportamiento organizacional positivo, es decir, con un estado positivo de desarrollo. Por su parte, Idil, Beliz \& Mehmet (2014), se refieren a él como el agregado de los recursos reales y potenciales que están vinculados a la posesión de una red duradera de relaciones institucionalizadas de conocimiento mutuo y el reconocimiento; sin embargo, reconocen que existen características de la organización desde la visión social del comportamiento, tales como: la confianza, las normas y redes que pueden mejorar la eficiencia de la organización. Según Delgado \& Castañeda (2011), para caracterizarlo es necesario referirse a cuatro dimensiones que le componen: autoeficacia, optimismo, esperanza y resiliencia.

La autoeficacia se entiende como las destrezas y riquezas cognitivas que posee un individuo, así como las capacidades necesarias para culminar exitosamente una tarea impuesta, además, tener confianza y autoeficacia para asumir y disponer del esfuerzo necesario para tener éxito en tareas desafiantes. Por otra parte, el optimismo se refiere a las atribuciones que posee el individuo acerca de éxitos actuales y futuros, por lo tanto, puede entenderse como la motivación que ayuda a que el individuo se esfuerce por realizar una tarea, implicando así que éste haga una atribución optimista y positiva sobre el futuro. La esperanza, es el estado emocional que permite al individuo empeñarse en conseguir un objetivo final, ser perseverante hacia la meta y tener la capacidad de reorientar los caminos hacia las metas diseñadas. Por último, la resiliencia es considerada como la capacidad que tiene el individuo de afrontar sucesos en la vida laboral que influyen significativamente y tomarlos como aprendizajes u oportunidades de mejora, es decir, a partir de los problemas y la adversidad, que sostienen la capacidad de recuperación a alcanzar el éxito (Delgado \& Castañeda, 2011; Contreras \& Juárez, 2013; Idil, 2015).

Citación del artículo: García, M., Forero, C. (2017). Elementos relacionales del capital psicológico y cambio organizacional en empleados del sector terciario de la cuidad de Bogotá - Colombia. Revista Katharsis, N 23, enero-julio 2017, pp.129-141, Disponible en http://revistas.iue.edu.co/index.php/katharsis 
Luthans et al. (2007) consideran el capital psicológico como un constructo de orden superior que representa la valoración positiva de las circunstancias y probabilidades de tener éxito basado en el esfuerzo. Por su parte, Fu Lam, Liang, Ashford \& Lee (2015), encontraron que las personas con un alto nivel de capital psicológico se caracterizan por tener un alto sentido de confianza, lo que aumenta la probabilidad de éxito en tareas difíciles (autoeficacia); además, estas personas tienden a: realizar atribuciones positivas para lograr tener éxito ahora y en el futuro (optimismo); la inclinación de algunos individuos a superar y perseverar en las metas, direccionando sus objetivos con el fin de tener éxito (esperanza); y, por último — cuando las personas son acosadas y se les dificulta realizar determinadas acciones por la adversidad - una tendencia a rebotar para alcanzar el éxito (resiliencia). Así pues, la relación entre capital psicológico y el rendimiento, es explicada indicando cuando las cuatro variables: eficacia, esperanza, el optimismo, y la resiliencia son combinadas para constituir el nivel de capital psicológico de una persona individual, permitiendo el logro del objetivo, la motivación y el éxito del resultado (Walumbwa, Peterson, Avolio \& Hartnell, 2010).

En consecuencia, el capital psicológico fomenta la participación de los empleados dentro de la organización; las personas que poseen las cuatro variables mencionadas anteriormente se caracterizan por tener mayor entrega y esfuerzo por culminar las metas propuestas dentro de la organización. Esta idea se contrasta con Luthans et al. (2007), el cual afirma que los cuatro factores del capital psicológico forman un orden en la construcción del rendimiento y la satisfacción de los individuos.

Un estudio realizado por Contreras \& Juárez (2013), tuvo como objetivo describir el capital psicológico de un grupo de directivos de PYMES colombianas y determinar si éste ejerce un efecto sobre sus prácticas de liderazgo. Las variables que se evaluaron en este estudio fueron los componentes del capital psicológico. Los resultados mostraron un alto nivel de capital psicológico en este grupo de directivos. En cuanto a la variables, la variable esperanza obtuvo la puntuación más alta, el segundo componente más importante fue la autoeficacia, la resiliencia, obtuvo una puntuación total de 150.2 la cual fue considerada alta de acuerdo con el instrumento utilizado (puntuación mayor a 147), finalmente se sugiere estudiar el rol del optimismo en el capital psicológico de este grupo de directivos, pues aunque no puede ser considerado bajo, fue una de las variables menos destacadas. Lo visto en este estudio el capital psicológico si influye en las prácticas de liderazgo, con esto se puede ver que los directivos de PYMES presentan un alto capital psicológico, siendo el principal componente la esperanza, como el más importante.

\section{Cambio organizacional}

Wustari, Dharmayati \& Permata (2015) señalan que el cambio organizacional es un fenómeno multifacético en la teoría de la organización contemporánea y lo definen como un proceso que se activa con la necesidad de generar un desarrollo en las organizaciones. Por otra parte, González, García \& Murillo (2014) sostienen que el cambio organizacional puede ser definido como el impacto dentro de las estructuras organizacionales, el poder y los altos niveles de la propiedad, procesos de decisión, la incorporación de nuevas tecnologías, organización del poder y cómo esto se acopla a las estructuras, así como la modificación en la forma, la calidad o el estado sobre el tiempo en una entidad organizativa (Steele, Celeste, Wilkinson \& Harvey, 2015). Por ello, las organizaciones actuales se describen como

Citación del artículo: García, M., Forero, C. (2017). Elementos relacionales del capital psicológico y cambio organizacional en empleados del sector terciario de la cuidad de Bogotá - Colombia. Revista Katharsis, N 23, enero-julio 2017, pp.129-141, Disponible en http://revistas.iue.edu.co/index.php/katharsis 
sistemas complejos que están en una continua búsqueda del equilibrio entre el orden y el caos.

En la actualidad cada organización debe ser consiente del incremento del mundo competitivo en el que se encuentra; es por esto que con el fin de cumplir las demandas externas debe implementar un programa de cambio organizacional (Wustari, Dharmayati \& Permata, 2015). En consecuencia, el cambio nace (y debe nacer) de la necesidad que tienen las organizaciones para hacer frente a las presiones ambientales y poder alcanzar los objetivos de supervivencia. Igualmente, se sostiene que el cambio es necesario para hacer frente a eventos inesperados en un entorno organizacional. Por esta razón, hablan del cambio como la práctica que provoca múltiples tensiones en múltiples direcciones, creando una complejidad particular.

De acuerdo con Jacobs, Witteloostuijn \& Zeyse (2013) en el cambio organizacional existen aspectos psicológicos de los seres humanos — actitudes, percepciones de cambio, estrategias de afrontamiento y cambios inducidos por el estrés - utilizados, por lo general, para cambiar a los individuos. Por ello, el estado de preparación organizacional, así como la implementación del cambio, deben ser respaldados con el compromiso y la confianza de los empleados.

Joaquiin, Santiago \& Carlos (2013), comentan los efectos que un proceso de cambio organizacional puede generar en las emociones de las personas, incidiendo en el comportamiento de los miembros de la organización. Por lo tanto, el cambio en todas las organizaciones se genera por un número de factores externos como la internacionalización y globalización, la exigencia de los consumidores, y la alta demanda de productos y servicios que los obliga a introducir nuevas tecnologías, provocando así la exigencia de un proceso de transformación.

Por su parte, Bass \& Steidlmeier (1999) consideran tres tipos de cambio: el cambio gradual, el de transición y el transformacional. El cambio gradual o de desarrollo se refiere a la mejora que la empresa hace en el momento actual de cambio (en lugar de crear algo nuevo). El cambio de transición reemplaza lo ya existente con algo completamente nuevo; y, por último, según Majid, Abdullah, Yasir \& Tabassum (2011), el cambio transformacional es un modelo que introduce nuevas y, radicalmente diferentes, formas de misión, cultura y liderazgo a la organización. Cabe señalar, además, que el cambio puede entenderse desde dos perspectivas: en primer lugar, el cambio como tal; las partes resaltantes se dan en cuanto a velocidad, secuencia y continuidad. En segundo lugar, la forma como se expresa organizacionalmente. Los temas resaltantes son cambio y aprendizaje, cambio visto desde las personas, procesos o grupos (Young, 2009).

De acuerdo a la manera como se maneje el cambio, se puede generar resistencia. Según Jungsik (2013) la resistencia es definida como la habilidad de una persona de rebotar del conflicto, la adversidad, el fracaso y la incertidumbre, llegando a captar el cambio seguro y la responsabilidad. Por otra parte, Knowles \& Linn (2004), sugieren que la resistencia se puede entender como una forma de retroalimentación para el agente de cambio. Es por esto que un supuesto fundamental en la teoría de la gestión de recursos humanos es el equilibrio entre el empleado y los intereses del empleador que se remonta a la distinción entre los objetivos personales y objetivos de la organización.

En muchos casos, la resistencia se percibe en el cambio organizacional como una amenaza y puede llegar a crear inseguridad en el empleo y amenazas en la autoestima individual y, de modo similar, en el bienestar de los individuos (Jungsik, 2013).

Citación del artículo: García, M., Forero, C. (2017). Elementos relacionales del capital psicológico y cambio organizacional en empleados del sector terciario de la cuidad de Bogotá - Colombia. Revista Katharsis, N 23, enero-julio 2017, pp.129-141, Disponible en http://revistas.iue.edu.co/index.php/katharsis 
El estudio realizado por Beal, Stavros \& Cole (2013), tuvo como objetivo examinar el posible papel de la resistencia al cambio como un moderador de la relación predictiva entre capital psicológico y el comportamiento de ciudadanía organizacional, en la que el comportamiento de ciudadanía organizacional sirvió como un índice para medir el cambio organizacional. Se utilizó una muestra de 97 empleados de una organización gubernamental que se encarga de brindar apoyo a la gestión del ciclo de vida de carrera. Se administró mediante una encuesta en línea la cual constaba de tres instrumentos cuantitativos que se utilizan para medir el capital psicológico, resistencia al cambio y conducta organizacional ciudadana. En los resultados obtenidos se identificaron tres temas relacionados con el cambio organizacional y tres temas relacionados con la resistencia al cambio, cambio orientado a la transformación, cambio centrado en las personas y cambio estructural. En la resistencia al cambio se identificaron; resistencia afectiva, resistencia conductual y resistencia intencional cognitiva. Se obtuvo en el análisis cuantitativo que existen altos niveles de resistencia al cambio que modero el efecto positivo de capital psicológico sobre el comportamiento de la ciudadanía organizacional, por otro lado, el análisis temático revelo que las formas frecuentes de resistencia al cambio son afectivas, conductuales y cognitivas.

Uno de los referentes empíricos tomados para la realización de este estudio fue el desarrollado por Luthans et al. (2007), donde estudia la calidad del recurso psicológico para usar las variables como un indicador para los resultados organizacionales, estas han sido combinadas para moldear el capital psicológico. Para los autores, el capital psicológico hace referencia al estado psicológico seguro del desarrollo de una persona, la eficacia de tener éxito en tareas estimulantes, el optimismo de lo que sucederá ahora y en el futuro, la esperanza de que los objetivos darán resultado y la resiliencia sostenida de tener éxito, por lo tanto, se ha demostrado que estas cuatro dimensiones son conceptualmente independientes.

El impacto social que tendrá esta investigación es generar aportes teóricos y empíricos sobre la relación de las variables objeto de estudio y explicaciones que aporten a los organizaciones colombianas la forma como se maneja un cambio adecuado de cambio teniendo en cuenta algunas variables del individuo.

Este estudio busca determinar la relación entre el cambio organizacional y el constructo capital psicológico con cada uno de sus cuatro componentes en trabajadores de diferentes empresas de la ciudad de Bogotá. Como objetivos específicos se plantea: a. Identificar la disposición al cambio en trabajadores de diferentes empresas de la ciudad de Bogotá; b. Identificar la relación entre cambio organizacional y optimismo de la empresa; c. Identificar la relación entre cambio y esperanza de la empresa; d. Identificar la relación cambio y resiliencia de la empresa; y e. Identificar la relación cambio y auto-eficacia de la empresa.

\section{Método}

\section{Diseño}

Es una investigación no experimental bajo un estudio transversal correlacional, ya que pretende la cuantificación y medición de las variables a trabajar para calcular el índice de relación entre ellas y determinar el grado de relación entre las variables en un momento del tiempo específicamente (Hernández, Fernández \& Baptista, 2010). El empleo de este tipo de estudio se justifica dado que se busca establecer la relación entre las variables sin que sea Citación del artículo: García, M., Forero, C. (2017). Elementos relacionales del capital psicológico y cambio organizacional en empleados del sector terciario de la cuidad de Bogotá - Colombia. Revista Katharsis, N 23, enero-julio 2017, pp.129-141, Disponible en http://revistas.iue.edu.co/index.php/katharsis 
necesario establecer un grado de causalidad entre ellas, para esta investigación se busca determinar la relación entre capital psicológico (variable predictora) y cambio organizacional (variable criterio).

\section{Participantes}

La muestra fue no probabilística, en organizaciones que se encontraban pasando por lo menos por un proceso de cambio de primer orden. Los participantes fueron 100 empleados de diferentes empresas pertenecientes al sector privado y público de la ciudad de Bogotá. Las características de inclusión de la población fueron: personas vinculadas laboralmente en la empresa superior a seis meses y la aceptación voluntaria para participar en la investigación.

Esta muestra estuvo compuesta en un 58\% por mujeres y el $42 \%$ hombres, el $56.75 \%$ de los participantes eran solteros. En cuanto a la escolaridad, el $41.7 \%$ de la muestra posee educación secundaria. Por último, de acuerdo a la modalidad de contratación, el $67.7 \%$ posee un contrato a término indefinido, siendo este aspecto el más representativo.

\section{Procedimiento}

Se contacta con las empresas del sector público y privado de la ciudad de Bogotá, quienes permitieron realizar la investigación dentro de su organización. Se explica el consentimiento informado y la confidencialidad a los participantes y se procede a la aplicación de los instrumentos cuya duración es de aproximadamente $45 \mathrm{~min}$. La participación de los colaboradores fue de carácter voluntario y anónimo.

\section{$\underline{\text { Instrumentos }}$}

Para llevar a cabo la investigación se empleó 2 instrumentos, el primero para medir el cambio organizacional, es un instrumento que fue diseñado para tal fin por García \& Forero (2010). El instrumento está constituido por nueve variables principales, tres factores de primer orden y dos factores de segundo orden obtenidos a partir de múltiples procesos de análisis y que se exponen a continuación: 1. Variables principales: a) adaptabilidad; b) liderazgo; c) participación; d) comunicación; e) trabajo en equipo; e) aceptación; f) resistencia al cambio; g) conocimiento de la organización; h) cultura de cambio; 2. Factores de Primer orden: a) factor Individual; b) factor grupal y c) factor; 3. Factores de Segundo orden: a) factor I: gestión del cambio; b) factor II: proyección y desarrollo del cambio. La confiabilidad de la prueba es de 0.84 . La puntuación máxima en cada componente es 4 y la mínima 1.

Para medir capital psicológico se utilizó el instrumento para evaluar capital psicológico en las organizaciones colombianas IPSICAP diseñado y validado por Delgado (2013). El instrumento contiene 36 ítems, 9 para cada uno de los componentes del capital psicológico: esperanza, optimismo, resiliencia y auto-eficacia. La escala de respuesta es tipo Likert con seis opciones de respuesta, los cuales la persona debe marcar según su grado de vivencia respecto al ítem. El Alpha de Cronbach para cada una de las escalas: esperanza (0.83), optimismo (0.69), resiliencia (0.69) y auto-eficacia $(0.74)$.

\section{Aspecticos éticos}

Citación del artículo: García, M., Forero, C. (2017). Elementos relacionales del capital psicológico y cambio organizacional en empleados del sector terciario de la cuidad de Bogotá - Colombia. Revista Katharsis, N 23, enero-julio 2017, pp.129-141, Disponible en http://revistas.iue.edu.co/index.php/katharsis 
En la presente investigación se tuvo en cuenta los siguientes aspectos teniendo en cuenta resolución No 008430 de 1993: a) consentimiento informado (artículo 6 del capítulo II), b) se considera una investigación sin riesgos (artículo 11 del capítulo II).

Por otra parte, según la ley 1090 que reglamenta el ejercicio del psicólogo, en su artículo 47 refiere que el psicólogo tendrá el cuidado necesario en la presentación de resultados diagnósticos y demás inferencias basadas en la aplicación de pruebas, hasta tanto estén debidamente validadas y estandarizadas.

\section{Resultados}

Para determinar la relación entre cambio organizacional y el constructo capital psicológico, se aplicaron dos instrumentos donde se midieron cada una de las variables mencionadas en una muestra de 100 trabajadores de varias empresas de la ciudad de Bogotá, pertenecientes al sector económico secundario y terciario. Los resultados se presentan en dos fases: a) estadísticos descriptivos de los instrumentos de aplicación y prueba de normalidad de cambio y capital psicológico y b) correlación entre variables objeto de estudio.

\section{Estadísticos descriptivos de los instrumentos de aplicación}

Dentro de los estadísticos descriptivos del instrumento de aplicación se tuvo en cuenta las puntuaciones de la media, desviación típica, asimetría y curtosis obtenidas por medio del análisis de los datos en el software de SPSS. En la tabla 1 y 2, se evidencian los resultados obtenidos para cambio organizacional y capital psicológico.

Tabla 2.

Estadísticos descriptivos del cambio organizacional IMC

\begin{tabular}{|c|c|c|c|c|c|c|}
\hline & & & & \multicolumn{3}{|c|}{ Prueba de normalidad } \\
\hline & Media & $\begin{array}{l}\text { Desviación } \\
\text { Típica }\end{array}$ & Asimetría & Curtosis & $\mathrm{P}$ & Decisión \\
\hline Adaptabilidad & 3,2350 &, 58580 & $-1,076$ & 1,824 & 0,83 & $\begin{array}{c}\text { Retener la } \\
\text { hipótesis nula }\end{array}$ \\
\hline Aceptación & 3,0218 & ,40366 &,- 654 &, 818 &, 121 & $\begin{array}{c}\text { Retener la } \\
\text { hipótesis nula }\end{array}$ \\
\hline Comunicación & 3,1780 & ,67365 & $-1,231$ & 1,391 & 010 & $\begin{array}{r}\text { Rechazar la } \\
\text { hipótesis nula }\end{array}$ \\
\hline $\begin{array}{l}\text { Conocimiento de la } \\
\text { organización }\end{array}$ & 3,2150 & ,60351 &,- 811 & ,465 & .021 & $\begin{array}{l}\text { Rechazar la } \\
\text { hipótesis nula }\end{array}$ \\
\hline Cultura de cambio & 2,9383 & ,85794 &,- 746 &,- 262 &, 000 & $\begin{array}{l}\text { Rechazar la } \\
\text { hipótesis nula }\end{array}$ \\
\hline Gestión del cambio & 3,4051 & ,50019 & $-1,814$ & 5,147 &, 015 & $\begin{array}{c}\text { Rechazar la } \\
\text { hipótesis nula }\end{array}$ \\
\hline Liderazgo & 3,4437 &, 55417 &,- 999 & 4,139 & ,022 & $\begin{array}{c}\text { Rechazar la } \\
\text { hipótesis nula }\end{array}$ \\
\hline Nivel grupal & 3,1592 & 61641 & $-1,260$ & 1,802 & 057 & $\begin{array}{l}\text { Retener la } \\
\text { hipótesis nula }\end{array}$ \\
\hline Nivel individual & 3,3487 & ,49725 & $-1,365$ & 4,863 & ,162 & $\begin{array}{l}\text { Retener la } \\
\text { hipótesis nula }\end{array}$ \\
\hline Nivel organizacional & 2,8763 & ,39512 &, 103 &,- 732 & 403 & $\begin{array}{l}\text { Retener la } \\
\text { hipótesis nula }\end{array}$ \\
\hline $\begin{array}{l}\text { Participación } \\
\text { integración }\end{array}$ & 3,3430 & ,64362 & $-1,452$ & 1,873 & ,001 & $\begin{array}{c}\text { Rechazar la } \\
\text { hipótesis nula }\end{array}$ \\
\hline
\end{tabular}

Citación del artículo: García, M., Forero, C. (2017). Elementos relacionales del capital psicológico y cambio organizacional en empleados del sector terciario de la cuidad de Bogotá - Colombia. Revista Katharsis, N 23, enero-julio 2017, pp.129-141, Disponible en http://revistas.iue.edu.co/index.php/katharsis 


\begin{tabular}{|c|c|c|c|c|c|c|}
\hline $\begin{array}{l}\text { Proyección y } \\
\text { desarrollo del cambio }\end{array}$ & 3,0640 &, 70435 & $-1,106$ &, 753 &, 018 & $\begin{array}{r}\text { Rechazar la } \\
\text { hipótesis nula }\end{array}$ \\
\hline Resistencia al cambio & 2,6280 &, 75146 &,- 129 &,- 972 & ,394 & $\begin{array}{c}\text { Retener la } \\
\text { hipótesis nula }\end{array}$ \\
\hline Trabajo en equipo & 2,9033 & ,87431 &,- 699 &,- 225 &, 055 & $\begin{array}{c}\text { Retener la } \\
\text { hipótesis nula }\end{array}$ \\
\hline
\end{tabular}

P. Prueba Kolmogorov-smirnov de una muestra

Los resultados de la variable cambio organizacional son homogéneos, ya que la desviación estándar presenta valores similares; esto indica que está muy cerca de la media. Por otro lado, teniendo en cuenta la curtosis, asimetría, el Kolmogorov-Smirnov, se evidencia que la mayoría de las sub-dimensiones no presentan un comportamiento normal.

Ahora, con relación a las sub-dimensiones que presentan un comportamiento normal se observa una congruencia en las respuestas, siendo nivel individual la de mayor puntuación con una media de 3,3487 superior a 1, lo cual indica que existe una heterogeneidad en el grupo de datos; por otro lado, resistencia al cambio presenta una puntuación menor en la media 2,6280. De igual forma las desviaciones estándar son de valores pequeños, lo cual indica que los datos están concentrados muy cerca de la media.

Tabla 2.

Estadísticos descriptivos del capital psicológico

\begin{tabular}{|c|c|c|c|c|c|c|}
\hline & & & & \multicolumn{3}{|c|}{ Prueba de normalidad } \\
\hline & Media & $\begin{array}{l}\text { Desviación } \\
\text { Típica }\end{array}$ & Asimetría & Curtosis & $\mathrm{P}$ & Decisión \\
\hline Esperanza & 5,0108 & ,79177 & $-1,328$ & 2,366 & , 155 & $\begin{array}{c}\text { Retener la } \\
\text { hipótesis nula }\end{array}$ \\
\hline Optimismo & 4,5538 & ,68360 & $-1,598$ & 4,677 & ,207 & $\begin{array}{c}\text { Retener la } \\
\text { hipótesis nula }\end{array}$ \\
\hline Resiliencia & 4,7417 & ,68415 & $-1,062$ & 2,425 & ,217 & $\begin{array}{l}\text { Retener la } \\
\text { hipótesis nula }\end{array}$ \\
\hline $\begin{array}{l}\text { Auto- } \\
\text { eficacia }\end{array}$ & 4,5304 & ,78318 &,- 582 &,- 092 & ,082 & $\begin{array}{c}\text { Retener la } \\
\text { hipótesis nula }\end{array}$ \\
\hline
\end{tabular}

P. Prueba Kolmogorov-smirnov de una muestra

Los resultados obtenidos de la tabla 2, muestra que la esperanza fue el factor que puntuó alto en las respuestas de los participantes en cuanto a capital psicológico correspondiente a una media de 5,0108 y la desviación estándar o típica fue de 0,79177 lo que quiere decir que se acerca a la media. La auto-eficacia fue el factor que puntuo menos con una media de 4,5304. Según la desviación estándar o típica los datos obtenidos presentan valores pequeños que son representativos ya que se acercan a la media.

Las pruebas de normalidad muestran que las variables de cambio organizacional no presentan un comportamiento normal, sucediendo lo contrario en el capital psicológico, razón por la cual se decide aplicar el estadístico Rho de Sperman.

Tabla 4.

Correlaciones entre las variables de cambio organizacional y capital psicológico

\begin{tabular}{llccccc}
\hline & & & Esperanza & Optimismo & Resiliencia & Auto-eficacia \\
\hline Aceptación & $\begin{array}{l}\text { Coeficiente } \\
\text { correlación }\end{array}$ & de &, $341^{* *}$ &, $208^{*}$ &, $244^{*}$ &, $381^{* *}$ \\
\hline
\end{tabular}

Citación del artículo: García, M., Forero, C. (2017). Elementos relacionales del capital psicológico y cambio organizacional en empleados del sector terciario de la cuidad de Bogotá - Colombia. Revista Katharsis, N 23, enero-julio 2017, pp.129-141, Disponible en http://revistas.iue.edu.co/index.php/katharsis 


\begin{tabular}{|c|c|c|c|c|c|c|}
\hline Comunicación & $\begin{array}{l}\text { Coeficiente } \\
\text { correlación }\end{array}$ & de & ,098 &, $281^{* *}$ & ,061 & ,080 \\
\hline $\begin{array}{l}\text { Conocimiento de la } \\
\text { organización }\end{array}$ & $\begin{array}{l}\text { Coeficiente } \\
\text { correlación }\end{array}$ & de & ,219* & ,128 &, $300^{* *}$ &, 154 \\
\hline Cultura de cambio & $\begin{array}{l}\text { Coeficiente } \\
\text { correlación }\end{array}$ & de & ,035 &, $235^{*}$ &,- 016 &,- 049 \\
\hline $\begin{array}{l}\text { Gestión del cambio } \\
\text { (Factor de segundo } \\
\text { Orden) }\end{array}$ & $\begin{array}{l}\text { Coeficiente } \\
\text { correlación }\end{array}$ & de &, $384^{* *}$ &, $362^{* *}$ &, $306^{* *}$ &, $389^{* *}$ \\
\hline Liderazgo & $\begin{array}{l}\text { Coeficiente } \\
\text { correlación }\end{array}$ & de &, $221^{*}$ &, $432^{* *}$ & ,201* &, $270^{* *}$ \\
\hline Nivel Grupal & $\begin{array}{l}\text { Coeficiente } \\
\text { correlación }\end{array}$ & de & , 190 &, $324^{* *}$ & ,107 &, 171 \\
\hline Nivel Individual & $\begin{array}{l}\text { Coeficiente } \\
\text { correlación }\end{array}$ & de & ,228* &, $445^{* *}$ &, 173 & , 192 \\
\hline $\begin{array}{l}\text { Nivel } \\
\text { Organizacional }\end{array}$ & $\begin{array}{l}\text { Coeficiente } \\
\text { correlación }\end{array}$ & de &, $512^{* *}$ &, 106 &, $364^{* *}$ &, $487^{* *}$ \\
\hline $\begin{array}{l}\text { Participación } \\
\text { Integración }\end{array}$ & $\begin{array}{l}\text { Coeficiente } \\
\text { correlación }\end{array}$ & de &, $406^{* *}$ &, $338^{* *}$ &, $279^{* *}$ &, $377^{* *}$ \\
\hline $\begin{array}{l}\text { Proyección } \\
\text { desarrollo } \\
\text { cambio } \\
\text { Orden) }\end{array}$ & $\begin{array}{l}\text { Coeficiente } \\
\text { correlación }\end{array}$ & de &, 119 &, $234^{*}$ &, 030 & 084 \\
\hline $\begin{array}{l}\text { Resistencia al } \\
\text { cambio }\end{array}$ & $\begin{array}{l}\text { Coeficiente } \\
\text { correlación }\end{array}$ & de &, $384^{* *}$ &,- 061 & ,239* &, $415^{* *}$ \\
\hline Trabajo en equipo & $\begin{array}{l}\text { Coeficiente } \\
\text { correlación }\end{array}$ & de &, 059 &, $247^{*}$ &, 050 & ,012 \\
\hline Total & $\begin{array}{l}\text { Coeficiente } \\
\text { correlación }\end{array}$ & de &, $430^{* *}$ &, $274^{* *}$ &, $289^{* *}$ &, $427^{* *}$ \\
\hline
\end{tabular}

**. La correlación es significativa al nivel 0,01 (bilateral)

*. La correlación es significativa al nivel 0,05(bilateral)

Las variables de capital psicológico y cambio organizacional presentan un nivel de significación entre el 0,01 (99\%) y 0,05 (95\%). Las sub-variables de cambio organizacional que se relación al 0.01 con las sub - variables de capital psicológico son: aceptación con esperanza (Rho ,341) y autoeficacia (Rho ,381); comunicación con optimismo (Rho ,281); conocimiento de la organización con resiliencia (Rho ,300); gestión del cambio con esperanza (Rho ,384), optimismo (Rho ,362), resiliencia (Rho ,306) y autoeficacia (Rho ,389); liderazgo con optimismo (Rho ,432) y autoeficacia (Rho ,270); nivel grupal con optimismo (Rho ,324); nivel individual con optimismo (Rho ,445); nivel organizacional con resiliencia (Rho ,364) y autoeficacia (Rho ,487); participación integración con esperanza (Rho ,406), optimismo (Rho ,338), resiliencia (Rho ,279) y autoeficacia (Rho ,377); resistencia al cambio con esperanza (Rho,384) y autoeficacia (Rho ,415).

Las sub-variables de cambio organizacional que se relación al 0.05 con las sub variables de capital psicológico son: aceptación con optimismo (Rho, 208) y resiliencia (Rho ,244); conocimiento de la organización con esperanza (Rho ,219); cultura de cambio con optimismo (Rho ,235); liderazgo con esperanza (Rho ,221) y resiliencia (Rho ,201); nivel individual con esperanza (Rho, 228); proyección y desarrollo de cambio con optimismo (Rho ,234) y, por último, trabajo en equipo con optimismo (Rho ,247).

Citación del artículo: García, M., Forero, C. (2017). Elementos relacionales del capital psicológico y cambio organizacional en empleados del sector terciario de la cuidad de Bogotá - Colombia. Revista Katharsis, N 23, enero-julio 2017, pp.129-141, Disponible en http://revistas.iue.edu.co/index.php/katharsis 


\section{Discusión}

Los resultados de esta investigación permitieron confirmar las seis hipótesis planteadas inicialmente en el marco del objetivo planteado, donde se propone determinar la relación entre cambio organizacional y el constructo capital psicológico teniendo en cuenta sus cuatro componentes.

Según el descriptivo de capital psicológico se evidencia que la variable esperanza fue el factor que puntuó más alto con una media de 5,0108, lo que se soporta en un estudio realizado por Fu Lam, Liang, Ashford \& Lee (2015), donde encontraron que las personas con un alto nivel de esperanza tienen la inclinación a superar y perseverar sus metas direccionando sus objetivos con el fin de tener éxito. Por otro lado, la variable auto-eficacia fue el factor que puntuó menos con una media de 4.5304, aunque la puntuación significativamente no es tan baja, si se presenta una diferencia en comparación a las otras variables. Tomando en cuenta el estudio ya mencionado las personas con alto nivel de autoeficacia tienen la probabilidad de tener más éxito en las tareas difíciles, lo que quiere decir que a las empresas a las que se les aplico el instrumento muestran falencias en la ejecución de las tareas.

Tomando en cuenta el análisis de resultados, la variable cambio organizacional, se comporta de manera homogénea, aunque la mayoría de las sub dimensiones no presentan un comportamiento normal. Esto sugiere, como lo menciona Louise (2014), que para las organizaciones sería favorable identificar en sus empleados estas características y ubicarlos en cargos que sirvan de apoyo a la organización para una adecuada adaptación en momentos de cambio.

Considerando los planteamientos de la investigación se puede determinar que el cambio organizacional y el capital psicológico mantienen una relación, ya que el cambio organizacional genera impacto dentro de las estructuras organizacionales como; el poder, los altos niveles de la propiedad, procesos de decisión, la incorporación de nuevas tecnologías y cómo estos se acoplan a las estructuras (González, García \& Murillo, 2014). Por otra parte, los cuatro factores del capital psicológico forman un orden en la construcción del rendimiento y la satisfacción de los individuos dentro de las organizaciones (Luthans et al., 2007).

En cuanto a los objetivos específicos, se evidencia en los resultados que existe una disposición al cambio organizacional en los trabajadores de diferentes empresas de la ciudad de Bogotá. Esto lo corroboran Mellert, Scherbaum \& Oliveira (2015), quienes afirman que para que se presente una disposición al cambio se debe instaurar en unas creencias y actitudes hacia un principio, encaminado hacia la facilidad que tiene el individuo de innovar y realizar cambios significativos tangibles para la organización; desde esta perspectiva, el cambio está diseñado para aumentar el rendimiento organizacional, mejorando la adaptación al ambiente y la preparación para futuros cambios.

Con respecto a las hipótesis se aceptan; H1: A mayor capital psicológico mayor será la disposición al cambio organizacional, H2: A mayor nivel de esperanza mayor será la disposición al cambio organizacional, H3: A mayor nivel de optimismo mayor será la disposición al cambio organizacional, H4: A mayor nivel de auto-eficacia mayor será la disposición al cambio organizacional y H5: A mayor nivel de resiliencia mayor será la disposición al cambio organizacional. Estas hipótesis, reafirman la relación entre capital psicológico y cambio organizacional. Esto lo contrastan Luthans et al. (2007), quien afirman que el capital psicológico podría incluso convertirse en un factor de sostenibilidad para lograr una ventaja competitiva en la economía global actual. Para estos autores, el capital Citación del artículo: García, M., Forero, C. (2017). Elementos relacionales del capital psicológico y cambio organizacional en empleados del sector terciario de la cuidad de Bogotá - Colombia. Revista Katharsis, N 23, enero-julio 2017, pp.129-141, Disponible en http://revistas.iue.edu.co/index.php/katharsis 
psicológico constituye una nueva dimensión de los recursos humanos y el cambio organizacional. Se considera el capital psicológico como una dimensión más, ya que esta tendrá importantes implicaciones; en cuanto a que involucra capacidades psicológicas susceptibles de ser medidas y desarrolladas para favorecer el cambio organizacional.

En concordancia con lo anterior, Avey, Luthans \& Jensen (2009) aseguran que los trabajadores con bajo nivel de capital psicológico, se muestran más despreocupados, tienen mayor tendencia a abandonar el cargo y suelen presentar más conductas disruptivas que afectan sus relaciones interpersonales. Es por esto que se evidencia que a mayor capital psicológico los trabajadores de las empresas tendrán un mejor desempeño en todas las áreas de la organización.

En la hipótesis 6, se encontró una correlación positiva entre los cuatro componentes del capital psicológico. Se acepta esta hipótesis de acuerdo al análisis de los resultados, en el cual se evidencia que existe una relación bidireccional entre la esperanza, el optimismo, la resiliencia y la autoeficacia en función del cambio organizacional de las empresas, generando así una relación estrecha y complementaria con la productividad. Esta idea se contrasta con la afirmación de Luthans et al. (2007), donde el capital psicológico fomenta la participación de los empleados dentro de la organización; las personas que poseen las cuatro variables, mencionadas anteriormente, se caracterizan por tener mayor entrega y esfuerzo por culminar las metas propuestas dentro de la organización.

Con este estudio se crea la necesidad de realizar investigaciones que tengan como propósito confirmar y confrontar los hallazgos, buscando plantear estudios que puedan determinar la causalidad de las variables y si este tiene algún componente determinante en la transformación de toda una cultura organizacional, foco que hasta ahora sigue siendo superfluo y carente de investigaciones argumentativas de tipo cuantitativo y cualitativo.

De los problemas relevantes que se presentaron durante el desarrollo de la investigación fue la falta de compromiso de la empresa elegida inicialmente ante la oportunidad para llevar a cabo la aplicación de los dos instrumentos, los argumentos de los trabajadores era la falta de compromiso y el trato inadecuado que reciben por parte de los gerentes y líderes de la misma. Fue así como se re direcciono la elección de la muestra poblacional a otras empresas de sector público y privado.

Con esto se puede concluir que en el contexto organizacional toma fuerza la tendencia a investigar las relaciones entre las variables de capital psicológico, cambio organizacional y cómo estas influyen de forma positiva o negativa en el desempeño laboral, esto permitirá descubrir, explorar y determinar fortalezas humanas que estructuran y mueven a las organizaciones; así como la relación de las instituciones públicas y privadas que llevan a cabo el proceso de cambio organizacional.

Esta investigación se convierte en parte de un cuerpo teórico que continúa nutriéndose sobre cambio organizacional, fundamentada en la gestión del capital psicológico que se proyecta a establecer relaciones causales a futuro, como punto de partida para construir estudios que lleven a los nuevos investigadores a determinar la magnitud y los alcances que pueden tener los factores psicológicos al interactuar con la visión de la transformación del cambio organizacional.

\section{Referencias}

Citación del artículo: García, M., Forero, C. (2017). Elementos relacionales del capital psicológico y cambio organizacional en empleados del sector terciario de la cuidad de Bogotá - Colombia. Revista Katharsis, N 23, enero-julio 2017, pp.129-141, Disponible en http://revistas.iue.edu.co/index.php/katharsis 
Avey, J. B., Luthans, F., Jensen, S. M. (2009). Psychological capital: A positive resource for combating employee stress and turnover. Human Resource Management, 48, 677-693. doi:10.1002/hrm.20294

Bass, B. M., Steidlmeier, P. (1999). Ethics, character, and authentic transformational leadership behavior. Leadership Quarterly, 10, 181-217. doi: 10.1016/S10489843(99)00016-8

Beal, I., Stavros, J. M., Cole, M. L. (2013). Effect of psychological capital and resistance to change on organizational citizenship behavior. Journal of Industrial Psychology, 39(2), 1-11. doi: 10.4102/sajip.v39i2.1136

Contreras, F., Juárez, F. (2013). Efecto del capital psicológico sobre las prácticas de liderazgo PYMES colombianas. Revista Venezolana de Gerencia, 18 (62), 247-264. Recuperado de http://www.redalyc.org/articulo.oa?id=29026923007

Delgado, L.E., Castañeda, D.I. (2011). Relación entre capital psicológico y la conducta de compartir conocimiento en el contexto del aprendizaje organizacional. Acta Colombiana de Psicología, 14 (1), 61-70. Recuperado de http://www.redalyc.org/articulo.oa?id=79822602006

Delgado, L. (2013). La evaluación del capital psicológico en las organizaciones (capítulo 2). En: Delgado, L. y Vanegas, M. (Editoras) (2013). Psicología Organizacional: perspectivas y avances. Bogotá: Ecoe ediciones.

Fu Lam, C., Liang, J., Ashford, S., Lee, C. (2015). Job Insecurity and Organizational Citizenship Behavior: Exploring Curvilinear and Moderated Relationship. Journal of Applied Psychology, 100(2), 499-510. doi:10.1037/a0038659

García, M., Forero, C. (2010). IMC - Instrumento de Medición de Cambio Organizacional. Manual Técnico. Bogotá, Colombia: RH\&T. (Documento no publicado).

González, C., García, M., Murillo, G. (2014). Cambio institucional y organizacional en el sector financiero colombiano. Innovar, 24 (54), 27-42. doi:10.15446/innovar.v24n54.46428

Hernández, R., Fernández, C., Baptista, P. (2010). Metodología de la Investigación. México: McGraw Hill

Idil, F., Beliz, D., Mehmet, S. (2014). Unorthodox forms of capital in organizations: positive psychological capital, intellectual capital and social capital. Procedia-Social and Behavioral Sciences, 152 (1), 963-972. doi:10.1016/j.sbspro.2014.09.351

Idil, T. (2015). The Effect of Positive Psychological Capital on Emotional Labor. Business and Finance: Society for the study, 4 (2), 20-34. Recuperado de http://www.ssbfnet.com/ojs/index.php/ijrbs/article/view/23/23

Jacobs, G., Witteloostuijn, A., Zeyse, J. (2013). A theoretical framework of organizational change. Journal of Organizational Change Management, 26 (5),772-792. doi: /10.1108/JOCM-09-2012-0137

Joaquiin, R., Santiago, M., Carlos, C. (2013). Sobre el cambio organizacional. Una revisión bibliográfica. Innovar, $23 \quad(50), \quad 35-\quad 52 . \quad$ Recuperado de http://www.redalyc.org/articulo.oa?id=81828692004

Jungsik, K. (2013). Organizational change: and employee organizational identification: mediation of perceived uncertainty. Social Behavior And Personality, 41 (6), 10191034. doi: 10.2224/sbp.2013.41.6.1019

Citación del artículo: García, M., Forero, C. (2017). Elementos relacionales del capital psicológico y cambio organizacional en empleados del sector terciario de la cuidad de Bogotá - Colombia. Revista Katharsis, N 23, enero-julio 2017, pp.129-141, Disponible en http://revistas.iue.edu.co/index.php/katharsis 
Knowles, E. S., Linn, J. A. (2004). The importance of resistance to persuasion. In E. S. Knowles \& J. A. Linn (Eds.). Resistance and persuasion. Mahwah, NJ: Lawrence Erlbaum Associates.

Louise, B. (2014). Employee objections to organizational change: a framework for addressing management responses. Organization development journal, 1 (41), 41-54. Recuperado de http://lup.lub.lu.se/record/4147288

Luthans, F., Avolio, B. J., Avey, J. B., Norman, S. M. (2007). Positive psychological capital: Measurement and relationship with performance and satisfaction. Personnel Psychology, 6 (1), 541-572. doi: 10.1111/j.1744-6570.2007.00083.

Majid, A., Abdullah, M., Yasir, M., Tabassum, N. (2011). Organizational inertia and change portfolio: An analysis of the organizational environment in devloping countries. African Journal of Business Management, 5 (2). 383-388. doi: 10.5897/AJBM10.368

Mellert, L., Scherbaum, C., Oliveira, J. (2015). Examining the relationship between organizational change and financial loss. Journal of Organizational Change Management, 28 (1), 71-59. doi: 10.1108/JOCM-11-2013-0236.

Steele, C., Wilkinson, A., Celeste, A. Wilkinson, K., Harvey, M. (2015). Building resilience and organizational readiness during healthcare facility redevelopment transitions: Is it possible to thrive? Health Environments Research \& Design Journal, 9 (1), 10-33. doi: $10.1177 / 1937586715593552$

Walumbwa, F., Peterson, S., Avolio, B., Hartnell, C. (2010). An investigation of the relation of the relationships among leader and follower psychological capital, service climate, and job performance. Personnel Psychology, 63 (4), 937-635. doi :10.1111/j.1744570.2010 .01193 .

Wustari, L., Dharmayati, B., Permata, W. (2015). The role of leadership and employee's condition on reaction to organizational change. Social and Behavioral Sciences, 172 (1), 471-478. doi: 10.1016/j.sbspro.2015.01.385.

Young, M. (2009). A meta model of change. Journal of Organizational Change Management, 22 (5), 524-548. doi:10.1108/09534810910983488

Citación del artículo: García, M., Forero, C. (2017). Elementos relacionales del capital psicológico y cambio organizacional en empleados del sector terciario de la cuidad de Bogotá - Colombia. Revista Katharsis, N 23, enero-julio 2017, pp.129-141, Disponible en http://revistas.iue.edu.co/index.php/katharsis 